\title{
Network-Decomposed Hierarchical Cooperation in Ad Hoc Networks With Social Relationships
}

\author{
Cheol Jeong, Member, IEEE, and Won-Yong Shin, Senior Member, IEEE
}

\begin{abstract}
In this paper, we introduce a network-decomposed hierarchical cooperation (HC) protocol and completely characterize the corresponding throughput-delay trade-off for a large wireless ad hoc network formed in the context of social relationships. Instead of randomly picking source-destination pairings, we first consider a distance-based social formation model characterized by the social group density $\gamma$ and the number of social contacts per node, $q$, where the probability that any two nodes in distance $d$ away from each other are socially connected is assumed to be proportional to $d^{-\gamma}$, which is a feasible scenario. Then, using muiltihop and network-decomposed HC protocols under our social formation model, we analyze a generalized throughput-delay trade-off according to the operating regimes with respect to parameters $\gamma$ and $q$ in both a dense network of unit area and an extended network of unit node density via a non-straightforward network transformation strategy. Our main results reveal that as $\gamma$ increases, performance on the throughput-delay trade-off can remarkably be improved, compared to the network case with no social relationships. It is also shown that in the dense network, the network-decomposed HC protocol always outperforms the multihop protocol, while the superiority of the network-decomposed HC depends on $\gamma$ and the path-loss exponent in the extended network.
\end{abstract}

\section{Index Terms}

Ad hoc network, multihop (MH), network-decomposed hierarchical cooperation (HC), scaling law, social relationships, throughput-delay trade-off.

The work of W.-Y. Shin was supported by the Basic Science Research Program through the National Research Foundation of Korea (NRF) funded by the Ministry of Education (2017R1D1A1A09000835). The work of C. Jeong was supported by the NRF through the Korea Government under Grant NRF-2017R1C1B1009145. (Corresponding author: Won-Yong Shin.)

C. Jeong is with the School of Intelligent Mechatronics Engineering, Sejong University, Seoul 05006, Republic of Korea. E-mail: cheol.jeong@ieee.org.

W.-Y. Shin is with the Department of Computer Science and Engineering, Dankook University, Yongin 16890, Republic of Korea. E-mail: wyshin@dankook.ac.kr.

Manuscript received September 24, 2017; revised June 16, 2018; accepted August 21, 2018. 


\section{INTRODUCTION}

Communications between users (i.e., a source and a destination) over a wireless network usually take place based on friendship, which is defined as online or offline social relationships among users. In other words, a source and its destination(s) are not just randomly paired in real-world communications, and rather a source tends to select its destination(s) along with friendships. In [1]-[5], it was observed that social interactions among users indeed depend heavily on the geographic proximity of them. In [4], a close relationship between geographic distance and probability distribution of friendship was demonstrated by experimental results based on the LiveJournal social network. More specifically, it was shown that the probability of befriending a particular user is inversely proportional to the positive power of the geographic distance [4]. In [5], the degree of friendship related to the issue of space was further studied on Twitter - the number of friends according to distance follows a double power-law distribution on Twitter, indicating that the probability of befriending a particular Twitter user is significantly reduced beyond a certain geographic distance between users. Moreover, there have been extensive studies on understanding the nature of friendships with respect to the geographic distance in large-scale online social networks such as Twitter [6], Facebook [7], Flickr [8], LiveJournal [4], and Foursquare [9], while validating the smallworld phenomenon and scale-free degree distributions. On the other hand, it has widely been known that social relationships influence users' interactions with each other in physical space; thus, users' social ties are closely related to the interactions of users' communication devices subject to diverse physical coupling (see, e.g., [10], [11] and references therein). For example, users at close proximity in a social group can share their pictures and videos, play games with friends, or exchange files using device-to-device (D2D) communication [12]. The D2D communications between firefighters or between police officers for public safety are another examples of wireless social networks in which users are socially tied and their geographic distances are short. Another application of wireless social networks includes content-centric (caching) communications [13], [14] that content objects are cached by numerous nodes over a network, in which each request is served by nearby content source nodes in a friendship relation. For this reason, there has been a growing interest in analyzing the impact of social groups on the performance of wireless networks. In [12], [15]-[17], traffic offloading, resource allocation, and medium access control (MAC) protocols were designed for device-to-device wireless communications in a social-aware perspective. In [18], the impact of social selfishness on the performance of epidemic routing was also investigated in delay tolerant networks. Social context-aware small cell networks were designed in [19] by optimizing the overall allocation of resources. In [20], it was presented how to form multihop D2D connections based on a community-based approach in D2D communications. Moreover, the throughput scaling laws of large wireless ad hoc networks were also studied by incorporating the notion of social characteristics into their network models [21]-[24], where the throughput scaling results depend on the number of nodes and the geographic distance unlike the case with no social relationships. In this paper, we aim to characterize a fundamental throughput-delay trade-off of a large wireless ad hoc network, where users communicate with others in the context of social relationships.

\section{A. Related Work}

In [25], it was shown that the aggregate throughput of a large wireless network having $n$ source-destination (S-D) pairs randomly distributed in a unit area (i.e., a dense network) scales as $\Theta(\sqrt{n / \log n})$, which is achieved by the nearest-neighbor multihop (MH) protocol. 
This throughput scaling was improved to $\Theta(\sqrt{n})$ using percolation theory [26]. MH protocols were further studied and analyzed in various aspects [27]-[29]. There has been a great deal of research to improve the aggregate throughput of dense networks up to a linear scaling in [30][38]. It was shown that an almost linear throughput scaling, i.e., $\Theta\left(n^{1-\epsilon}\right)$ for an arbitrarily small $\epsilon>0$, can be achieved by hierarchical cooperation (HC) protocols [30], [31]. The impact and benefits of infrastructure support in improving the throughput scaling in hybrid networks, consisting of both wireless ad hoc nodes and infrastructure nodes, were studied in [32]-[34]. Novel techniques such as networks with node mobility [35] and directional antennas [36]-[38] were also introduced to achieve a linear throughput scaling. Besides the throughput, delay is also a key performance metric in most wireless network applications. One can usually improve the per-node throughput at the cost of an increased delay of a packet. The trade-off between throughput and delay metrics of both static and mobile ad hoc networks was examined in terms of scaling laws in some papers [39]-[42]. In [39], the throughput-delay trade-off of a mobile ad hoc network adopting a two-hop relay protocol was analyzed under a simple independent and identically distributed (i.i.d.) mobility model. In [40], [41], the throughput-delay trade-off was derived in another mobile network adopting a random walk mobility model as well as in a static network. In [42], the throughput-delay trade-off of a static ad hoc network was studied by modifying the original HC protocol in [30] in order to improve the delay performance for the same throughput.

In all these previous studies, it was assumed that a source selects its destination at random for analytical convenience. In practice, however, this assumption is hardly realistic since a source and its destination tend to be paired up along with one-to-one friendship in the presence of social groups. Thus, existing achievable schemes and analytical frameworks that show the capacity scaling laws cannot be directly applicable to the performance analysis of ad hoc networks with social groups. Recently, the notion of social relationships was taken into account in studying the capacity scaling laws of large wireless ad hoc networks [21]-[24]. In [21], the throughput scaling achieved by the $\mathrm{MH}$ protocol was analyzed again under a social formation model such that each node has a social group consisting of a fixed number of nodes and selects its destination uniformly among the nodes in its social group. This result was generalized in [22] by assuming social groups with different numbers of nodes and a non-uniform probability of selecting one destination in each social group. As an alternative approach to analyzing the network throughput scaling, it was assumed in [23] that the number of friends (i.e., the friendship degree) follows a Zipf's distribution [43] and the probability of befriending a particular user depends on both the geographic distance between nodes and the node density. In [24], the capacity scaling of a hybrid network with social contact behavior was also investigated.

\section{B. Main Contributions}

In this paper, we introduce a new HC protocol, termed a network-decomposed $\mathrm{HC}$ protocol, and characterize a general throughput-delay trade-off in a large wireless ad hoc network formed in the context of social relationships. To this end, we first consider a distancebased social formation model parameterized by the social group density $\gamma$, the number of social contacts per node, $q$, and the probability that a source selects its destination among social contacts. To better illustrate our results, we identify three operating regimes partitioned according to the social group density $\gamma$. More specifically, we focus on designing the networkdecomposed $\mathrm{HC}$ protocol so that the network under our social formation model operates properly, since the conventional HC protocols in [30], [42] do not guarantee the best tradeoff performance under our network model. In the proposed protocol, the whole network is 
divided into multiple non-overlapping subnetworks, each of which operates in parallel along with the HC protocol. A time-division multiple access (TDMA) operation is used to avoid an edge node problem that may occur with network decomposition. To be specific, when the network is decomposed into multiple subnetworks, there may exist some sources in a subnetwork, whose destinations are out of the subnetwork. To solve this problem, we apply the 4-TDMA strategy with which all the subnetworks are shifted to the right, shifted up, and shifted diagonally in consecutive three time slots and remain unshifted in one time slot. Using both the $\mathrm{MH}$ protocol in [21], revisited in the context of social relationships, and the network-decomposed $\mathrm{HC}$ protocol and then computing the average distance between a source and its destination derived under the social formation model, we completely characterize the throughput-delay trade-off according to each operating regime with respect to parameters $\gamma$ and $q$ in not only a dense network of unit area but also an extended network of unit node density through a non-straightforward network transformation strategy in terms of $\gamma$. As main results, we show that the throughput-delay trade-off is significantly improved as $\gamma$ increases beyond a certain value, compared to the network case with no social relationships. We also show that in the dense network, the network-decomposed HC protocol always outperforms the multihop protocol, while the superiority of the network-decomposed HC is determined according to $\gamma$ and the path-loss exponent in the extended network.

Our main contributions are five-fold and summarized as follows:

- We incorporate the notion of social relationships into a large wireless ad hoc network in order to analyze a fundamental throughput-delay trade-off for not only dense networks but also more challenging extended networks, whereas the throughput performance was only characterized in some prior studies dealing with wireless social networks [21] [24].

- Unlike the conventional HC protocols [30], [42] that did not exploit one-to-one social relationships, we propose a network-decomposed $\mathrm{HC}$ protocol that is properly designed based on the 4-TDMA strategy under the distance-based social formation model.

- To better interpret our results along with achievable schemes, we also identify three operating regimes on the throughput-delay trade-off with respect to the social group density and the number of social contacts.

- In both dense and extended networks with our social formation model, we completely characterize a general throughput-delay trade-off achieved by the $\mathrm{MH}$ and networkdecomposed $\mathrm{HC}$ protocols according to each operating regime.

- Furthermore, we conduct numerical evaluation to validate that our analytical results show trends consistent with computer simulation results.

\section{Organization and Notations}

The rest of the paper is organized as follows. The system model and preliminaries are given in Section III. The main results are presented in Section III] In Section IV, the networkdecomposed HC protocol is described and the corresponding throughput-delay trade-off is derived. In Section V numerical results are presented. Section VI summarizes the paper with some concluding remarks.

Throughout this paper, $\mathbb{E}[\cdot]$ indicates the statistical expectation; and $\operatorname{Pr}\{\mathcal{A}\}$ is the probability of an event $\mathcal{A}$. We use the following asymptotic notation: i) $f(x)=O(g(x))$ means that there exist positive constants $C$ and $c$ such that $f(x) \leq C g(x)$ for all $x>c$, ii) $f(x)=\Omega(g(x))$ if $g(x)=O(f(x))$, iii) $f(x)=\omega(g(x))$ means that $\lim _{x \rightarrow \infty} \frac{g(x)}{f(x)}=0$, and iv) $f(x)=\Theta(g(x))$ if $f(x)=O(g(x))$ and $g(x)=O(f(x))$. 


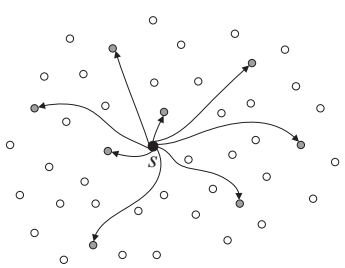

(a) $\gamma$ is small.

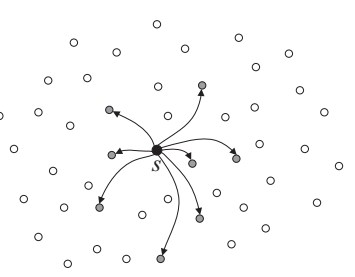

(b) $\gamma$ is large.

Fig. 1. The social group members (marked with shaded circles) of a source node $s$ when $q=8$.

\section{Models AND PROBlem Definition}

In this section, we first describe not only our network model but also our social formation model. We then present important lemmas that are required to derive our main results including the throughput-delay trade-off.

\section{A. System and Channel Models}

We consider the following two types of network configurations: 1) a dense network of unit area [25], [30], [40], [41] and 2) an extended network of unit node density [26], [30]. In what follows, the dense network model is assumed, and our main results will be extended to the extended network configuration later (refer to Sections III-B and IV-C). It is assumed that our network is composed of $n$ nodes, which are uniformly and independently distributed in a square of given unit area, and one central processor, which enables the network to be globally synchronized and coordinated. Specifically, the central processor plays a role of not only controlling interference based on the TDMA strategy but also determining packet delivery routes using geo-located information of nodes. Updated information is delivered to the central processor from nodes only when new S-D pairings are established. Each node acts as a source and has exactly one corresponding destination node. Now, let us turn to the channel modeling. Under wireless networks in light-of-sight (LOS) environments, the complex channel between nodes $i$ and $k$ is denoted by $h_{k i}=\frac{e^{j \theta_{k, i}}}{d_{k, i}^{\alpha / 2}}$ where $\theta_{k, i}$ is the random phase uniformly distributed in $[0,2 \pi) \cdot 1] d_{k, i}$ denotes the distance between nodes $i$ and $k$, and $\alpha \geq 2$ is the path-loss exponent. The phase $\theta_{k, i}$ and the path-loss model are based on a far-field assumption, i.e. the distance between any two nodes is assumed to be much larger than the carrier wavelength. It is also assumed that one-to-one friendship relations on online social networks are available at the central processor so that sources and destinations are paired up in the presence of social groups. Moreover, the central processor updates the social relationships between any two nodes, which takes place intermittently since the period of such changes to friendship would be much longer than the entire communication period.

\section{B. Social Formation Model}

In prior studies on the large-sale network analysis, each source node selects its destination node in a random fashion without taking into account the geographic distance between the

\footnotetext{
${ }^{1}$ In [44], it was shown that the capacity scaling of wireless networks is fundamentally limited by the relation between $n$ and the wavelength due to laws of physics. Based on this investigation, the throughput scaling achieved by HC was rediscovered in [45]. In this paper, however, we adopt the i.i.d. phase assumption as in [30], [31], [34] for analytical tractability. Moreover, we deal simply with the LOS channel rather than multipath fading channels to focus on analyzing the effect of social relationships in our network. This is due to the fact that throughput scaling laws do not fundamentally change in the presence of fading if all nodes have their own traffic demands [28], [29], [46], [47].
} 
two nodes, i.e., S-D pairings are randomly picked so that each node is the destination of exactly one source. It is thus likely that the source is far away from its destination. On the other hand, in our work, nodes are assumed to be geographically related with each other in a social context, which is more feasible in practice. In online social networks, it was observed that the probability of friendship formation (or social relationships) between two nodes (users) is proportional to the inverse of the power of the Euclidean distance between the two nodes (users) [3], [4]. Each node is allowed to have multiple friends by forming a social group for the node. A source node and its destination node are chosen only out of its social group members by the central processor that is aware of one-to-one friendship relations. In our distance-based social formation model, we assume that the Euclidean distance between a source and its social group members follows a power-law distribution as in [3], [4]. As illustrated in Fig. 1, a source node $s$ selects other node $v_{i}$ as its contact with a probability proportional to $d_{s, v_{i}}^{-\gamma}$, where $\gamma>0$ is the social group density. Each node has a social group that consists of $q$ contacts selected independently, where $q \in\{1, \cdots, n-1\}$ (i.e., $q=O(n)$ ). The probability that the set of nodes $\left\{v_{i_{1}}, \ldots, v_{i_{q}}\right\}$ forms a social group of the node $s$ is given by

$$
\operatorname{Pr}\left\{\text { Social group of } s=\left\{v_{i_{1}}, \ldots, v_{i_{q}}\right\}\right\}=\frac{d_{s, v_{i_{1}}}^{-\gamma} \cdots d_{s, v_{i_{q}}}^{-\gamma}}{N_{\gamma, q}},
$$

where $N_{\gamma, q}=\sum_{1 \leq i_{1}<\cdots<i_{q} \leq n} d_{s, v_{i_{1}}}^{-\gamma} \cdots d_{s, v_{i_{q}}}^{-\gamma}$ is the normalization factor. From (1), one can see that when the social group density $\gamma$ is large, the social group members of a node tend to be close to each other. After forming social groups, each source selects one of its social group members as its destination uniformly at random.

\section{Preliminaries}

In this subsection, we present important lemmas to analyze the throughput-delay tradeoff for the $\mathrm{MH}$ and network-decomposed $\mathrm{HC}$ protocols in the subsequent sections. In pure wireless ad hoc networks with no social group, since the sources and destinations are paired up one-to-one in a random fashion, the average distance between an S-D pair is given by $O(1)$ with high probability (w.h.p.) in a dense network. On the other hand, in the context of social relationships, the average distance of an S-D pair depends on both the number of contacts in a social group, $q$, and the social group density $\gamma$ since a source node selects one of its social contacts in the belonging social group as its destination node randomly. The average distance between an S-D pair is specified according to the parameters $q$ and $\gamma$ below.

Lemma 1: If $q$ scales faster than a constant independent of $n$ (i.e., $q=\omega(1)$ ), then the average distance between a source $s$ and its destination $v, \mathbb{E}\left[d_{s, v}\right]$, in a dense network is given by

$$
\mathbb{E}\left[d_{s, v}\right]=\Theta(1)
$$

If $q=\Theta(1)$, then $\mathbb{E}\left[d_{s, v}\right]$ in a dense network is given by

$$
\mathbb{E}\left[d_{s, v}\right]= \begin{cases}\Theta(1) & \text { for } 0 \leq \gamma<2 \\ \Theta\left(\left(\frac{\log n}{n}\right)^{\frac{\gamma}{2}-1}\right) & \text { for } 2 \leq \gamma \leq 3 \\ \Theta\left(\sqrt{\frac{\log n}{n}}\right) & \text { for } \gamma>3\end{cases}
$$

where $\gamma>0$ denotes the social group density. 
Proof: From [21, Section IV], when the MH protocol is employed in a dense network, the average number of hops in any given $S-D$ routing path, $\mathbb{E}[X]$, is shown to be

$$
\mathbb{E}[X]= \begin{cases}\Theta\left(\frac{1}{r(n)}\right) & \text { for } q=\omega(1) \\ \Theta\left(\frac{n}{n-q+1} \frac{1}{r(n)}\right) & \text { for } q=\Theta(1), 0 \leq \gamma<2 \\ \Theta\left(\frac{n}{n-q+1} \frac{1}{r^{3-\gamma(n)}}\right) & \text { for } q=\Theta(1), 2 \leq \gamma \leq 3 \\ \Theta\left(\frac{n}{n-q+1}\right) & \text { for } q=\Theta(1), \gamma>3,\end{cases}
$$

where $r(n)=\Theta\left(\sqrt{\frac{\log n}{n}}\right)$ is the transmission range per hop. By multiplying $\mathbb{E}[X]$ by $r(n)$, the average distance $\mathbb{E}\left[d_{s, v}\right]$ can be expressed as

$$
\mathbb{E}\left[d_{s, v}\right]= \begin{cases}\Theta(1) & \text { for } q=\omega(1) \\ \Theta\left(\frac{n}{n-q+1}\right) & \text { for } q=\Theta(1), 0 \leq \gamma<2 \\ \Theta\left(\frac{n}{n-q+1}\left(\frac{\log n}{n}\right)^{\frac{\gamma}{2}-1}\right) & \text { for } q=\Theta(1), 2 \leq \gamma \leq 3 \\ \Theta\left(\frac{n}{n-q+1} \sqrt{\frac{\log n}{n}}\right) & \text { for } q=\Theta(1), \gamma>3,\end{cases}
$$

which thus leads to (2) and (3). This completes the proof of this lemma.

Since the distance between two nodes in an extended network is simply increased by a factor of $\sqrt{n}$ compared to a dense network, the average distance in the extended network is also increased by a factor of $\sqrt{n}$.

A natural question arises to examine whether the distance between an S-D pair may deviate from its mean. In the following lemma, we show that the distance of an S-D pair does not scale faster than the average distance $\mathbb{E}\left[d_{s, v}\right]$ w.h.p.

Lemma 2: The distance between a source $s$ and its destination $v, d_{s, v}$, does not scale at a faster rate than the average distance $\mathbb{E}\left[d_{s, v}\right]$ within a factor of $n^{\epsilon}$ w.h.p. for an arbitrarily small $\epsilon>0$. That is, the probability $\operatorname{Pr}\left\{d_{s, v}<n^{\epsilon} \mathbb{E}\left[d_{s, v}\right]\right\}$ is given by $1-\frac{1}{n^{\epsilon}}$.

Proof: By Markov inequality 2 we have

$$
\begin{aligned}
\operatorname{Pr}\left\{d_{s, v}<n^{\epsilon} \mathbb{E}\left[d_{s, v}\right]\right\} & =1-\operatorname{Pr}\left\{d_{s, v} \geq n^{\epsilon} \mathbb{E}\left[d_{s, v}\right]\right\} \\
& \geq 1-\frac{\mathbb{E}\left[d_{s, v}\right]}{n^{\epsilon} \mathbb{E}\left[d_{s, v}\right]}=1-\frac{1}{n^{\epsilon}},
\end{aligned}
$$

which tends to one as $n$ goes to infinity. This completes the proof of this lemma.

Hence from Lemmas 1 and 2 , one can straightforwardly replace the average distance $\mathbb{E}\left[d_{s, v}\right]$ by the distance $d_{s, v}$ of a certain S-D pair as long as a factor of $n^{\epsilon}$ can be ignored.

\section{Performance Metrics}

In this subsection, we formally define the throughput and delay used throughout the paper.

Definition 1 (Throughput): A per-node throughput $R(n)$ is said to be achievable w.h.p. if all sources can transmit at the average rate of $R(n)$ bits $/ \mathrm{s} / \mathrm{Hz}$ to their destinations with probability approaching one as $n$ increases. The achievable aggregate throughput is given by $T(n)=\Omega(n R(n))$ accordingly.

\footnotetext{
${ }^{2}$ One can use a tighter inequality with the second moment of random variable $d_{s, v}$ (e.g., Chebyshev's inequality), which however does not fundamentally change our main results.
} 
Definition 2 (Delay): The end-to-end delay of a packet is the time that it takes for the packet to reach its destination after it leaves the source. The delay $D(n)$ is the expectation of the average delay over all $\mathrm{S}-\mathrm{D}$ pairs and is expressed as

$$
D(n)=\frac{2}{n} \sum_{i=1}^{n / 2} D_{i}(n),
$$

where $D_{i}(n)$ is the sample mean of delay (over packets that reach their destinations) for $\mathrm{S}-\mathrm{D}$ pair $i$.

Note that the queuing delay at the source node is not included in our work. Although the queuing delay may account for a large portion of the overall delay of a packet, it was shown in [41] that when the queuing delay is taken into account, the throughput-delay tradeoff for the $\mathrm{MH}$ protocol remains unchanged in order sense since the average delay at each server, corresponding to a routing cell, is bounded by some constant independent of $n$. Hence, Theorems 1 and 3 in Section III will remain the same even with the queuing delay. On the other hand, for the HC protocol, the analysis of delay scaling with the queuing delay is left open even under network models without social relationships.

\section{MAIN RESUlTS}

In this section, we present main results of this paper by characterizing the throughput-delay trade-off achieved by the MH and network-decomposed HC protocols with the distance-based social formation model in Section II-B. The throughput-delay trade-off depends on the pathloss exponent $\alpha$ and the parameters of the social formation model such as the social group density $\gamma$ and the number of contacts in a social group, $q$. To illustrate the main results more concisely, we will first identify operating regimes on the throughput-delay trade-off as follows:

- Regime A (Low social group density regime): $q=\omega(1)$ or $\{q=\Theta(1)$ and $0 \leq \gamma<2\}$

- Regime B (Medium social group density regime): $q=\Theta(1)$ and $2 \leq \gamma \leq 3$

- Regime $\mathbf{C}$ (High social group density regime): $q=\Theta(1)$ and $\gamma>3$.

That is, the entire operating regimes are categorized by $\gamma$ and $q$.

Note that similarly as in other studies on the throughput scaling laws of ad hoc networks [25]-[42], [45], [46], it is possible to have no outage event by globally controlling interference based on the TDMA operation among square routing cells. Thus, we do not employ any retransmission scheme in our case since we will make the outage probability approach zero. Next, we will briefly account for the $\mathrm{MH}$ protocol in ad hoc networks, which was originally introduced in [25] and then was generalized in [40]. The overall procedure of the $\mathrm{MH}$ protocol is described as follows:

- The network is divided into square routing cells of area $a(n)=\Omega(\log n / n)$ in a dense network $(a(n)=\Omega(\log n)$ in an extended network) ensuring that each cell includes at least one node w.h.p. [25], [40].

- Draw a line connecting a source to its destination and perform MH routing horizontally or vertically by using the adjacent routing cells passing through the line until its packet reaches the corresponding destination.

- By virtue of the central processor, each routing cell operates the $t$-TDMA to avoid huge interference, where $t>0$ is some small constant independent of $n$.

The network-decomposed HC protocol will be described in detail in Section IV-B. 


\section{A. Throughput-Delay Trade-off in Dense Networks}

In this subsection, we show the throughput-delay trade-off achieved by the $\mathrm{MH}$ and network-decomposed HC protocols in a dense network. The number of S-D lines passing through each cell in the MH protocol is first specified in the following lemma.

Lemma 3: For $a(n)=\Omega(\log n / n)$, the total number of S-D lines passing through each cell is $O\left(\mathbb{E}\left[d_{s, v}\right] n \sqrt{a(n)}\right)$, where $\mathbb{E}\left[d_{s, v}\right]$ is the average distance of an S-D pair.

Proof: By generalizing the arguments in [40, Lemma 3] to the case of an arbitrary average distance of an S-D pair, one can easily show that the total number of S-D lines passing through each cell is given by $O\left(\mathbb{E}\left[d_{s, v}\right] n \sqrt{a(n)}\right)$. This completes the proof of this lemma.

The main result achieved by the $\mathrm{MH}$ protocol in a dense network is shown in the following theorem.

Theorem 1: The throughput-delay trade-off for the $\mathrm{MH}$ protocol in a wireless dense network adopting the social formation model is given by

$$
(T(n), D(n))=\left\{\begin{array}{c}
\left(\Theta\left(\frac{1}{\sqrt{a(n)}}\right), \Theta\left(\frac{1}{\sqrt{a(n)}}\right)\right) \\
\text { for Regime A } \\
\left(\Theta\left(\left(\frac{n}{\log n}\right)^{\frac{\gamma}{2}-1} \frac{1}{\sqrt{a(n)}}\right), \Theta\left(\left(\frac{\log n}{n}\right)^{\frac{\gamma}{2}-1} \frac{1}{\sqrt{a(n)}}\right)\right) \\
\text { for Regime B } \\
\left(\Theta\left(\sqrt{\frac{n}{a(n) \log n}}\right), \Theta\left(\sqrt{\frac{\log n}{a(n) n}}\right)\right) \\
\text { for Regime C, }
\end{array}\right.
$$

where the area of each square cell is given by $a(n)=\Omega(\log n / n)$.

Proof: Using Lemmas 1 and 3, the number of S-D lines passing through each cell, $u(n)$, is given by

$$
u(n)= \begin{cases}O(n \sqrt{a(n)}) & \text { for Regime A } \\ O\left(\left(\frac{\log n}{n}\right)^{\frac{\gamma}{2}-1} n \sqrt{a(n)}\right) & \text { for Regime B } \\ O\left(\sqrt{\frac{\log n}{n}} n \sqrt{a(n)}\right) & \text { for Regime C. }\end{cases}
$$

Due to the fact that the total traffic through each cell is the traffic generated by all the S-D lines passing through the cell and the cell throughput is $\Theta(1)$ under the TDMA operation, the aggregate throughput $T(n)$ is lower-bounded by

$$
\begin{aligned}
T(n)= & \left(\frac{n}{u(n)}\right) \\
& = \begin{cases}\Theta\left(\frac{1}{\sqrt{a(n)}}\right) & \text { for Regime A } \\
\left.\Theta\left(\frac{n}{\log n}\right)^{\frac{\gamma}{2}-1} \frac{1}{\sqrt{a(n)}}\right) & \text { for Regime B } \\
\Theta\left(\sqrt{\frac{n}{\log n}} \frac{1}{\sqrt{a(n)}}\right) & \text { for Regime C. }\end{cases}
\end{aligned}
$$

We now turn to computing the average packet delay. Since each hop covers a distance of $\Theta(\sqrt{a(n)})$ and the delay is at most a constant times the number of hops, from Lemma 1, the 
delay $D(n)$ is given by

$$
\begin{aligned}
D(n)=\Theta\left(\frac{\mathbb{E}\left[d_{s, v}\right]}{\sqrt{a(n)}}\right) & \text { for Regime A } \\
& = \begin{cases}\Theta\left(\frac{1}{\sqrt{a(n)}}\right) & \text { for Regime B } \\
\left.\Theta\left(\frac{\log n}{n}\right)^{\frac{\gamma}{2}-1} \frac{1}{\sqrt{a(n)}}\right) & \text { for Regime C. } \\
\Theta\left(\sqrt{\frac{\log n}{n}} \frac{1}{\sqrt{a(n)}}\right) & \end{cases}
\end{aligned}
$$

Hence, using (5) and (6) finally leads to (4), which completes the proof of this theorem.

From the above result, the following observations can be found according to each operating regime. In Regime A (i.e., the low social group density regime), the throughput-delay tradeoff for the MH protocol is the same as that in [40], in which no social group exists. This is because small values of $\gamma$ correspond to the case where the social group members are widely distributed over the whole network and hence the average distance of an S-D pair is not reduced compared to the network case with no social group. In Regime $\mathrm{B}$, as $\gamma$ increases, the throughput is improved and the delay is reduced by virtue of the decreased average distance of an S-D pair. In Regime C, the maximum throughput $\Theta(n)$ can be achieved by only using $\Theta(1)$ hops. This is consistent with the throughput scaling result according to the social group density in [21].

Remark 1: In our dense network, the throughput-delay trade-off for the MH protocol is illustrated in Fig. 2 when $q=\Theta(1)$. The red arrows in Fig. 2 represent the throughput-delay scaling results achieved by the MH protocol according to different values of $\gamma$. More specifically, when $0 \leq \gamma<2$ (Regime A), the trade-off is given by $(T(n), D(n))=\left(\frac{1}{\sqrt{a(n)}}, \frac{1}{\sqrt{a(n)}}\right)$ for $a(n)=\Omega\left(\frac{\log n}{n}\right)$ and $a(n)=O(1)$. In Regime $\mathrm{B}$, as an exemplary value of $\gamma$, the tradeoff for $\gamma=\frac{5}{2}$ is illustrated in the figure for $a(n)=\Omega\left(\frac{\log n}{n}\right)$ and $a(n)=O\left(\frac{1}{\sqrt{n}}\right)$, where $(T(n), D(n))=\left(\Theta\left(\left(\frac{n}{\log n}\right)^{\frac{1}{4}} \frac{1}{\sqrt{a(n)}}\right), \Theta\left(\left(\frac{\log n}{n}\right)^{\frac{1}{4}} \frac{1}{\sqrt{a(n)}}\right)\right)$. When $\gamma>3$ (Regime C), the trade-off is given by $(T(n), D(n))=\left(\Theta\left(\frac{n}{\log n}\right), \Theta(1)\right)$ for $a(n)=\Theta\left(\frac{\log n}{n}\right)$, which corresponds to a single point in the figure. As seen in Fig. 2, the throughput-delay trade-off gets improved significantly as the social group density $\gamma$ increases.

Remark 2: To better understand the above trade-off between throughput and delay for the $\mathrm{MH}$ protocol in the dense network, we express $T(n)$ as a function of $D(n)$ as follows:

$$
T(n)= \begin{cases}\Theta(D(n)) & \text { for Regime A } \\ \Theta\left(D(n) n^{\gamma-2-\epsilon}\right) & \text { for Regime B } \\ \Theta\left(D(n) n^{1-\epsilon}\right) & \text { for Regime C }\end{cases}
$$

for an arbitrarily small $\epsilon>0$. It is again observed that the throughput $T(n)$ given the delay $D(n)$ can be greatly improved as $\gamma$ increases.

We modify the HC protocol in [42] so that the network under our social formation model operates properly, which will be explained in detail in Section IV-B. We herein summarize our main results for the proposed network-decomposed HC protocol when the notion of social relationships is incorporated into ad hoc networks. The main results for a dense network are shown as follows. 


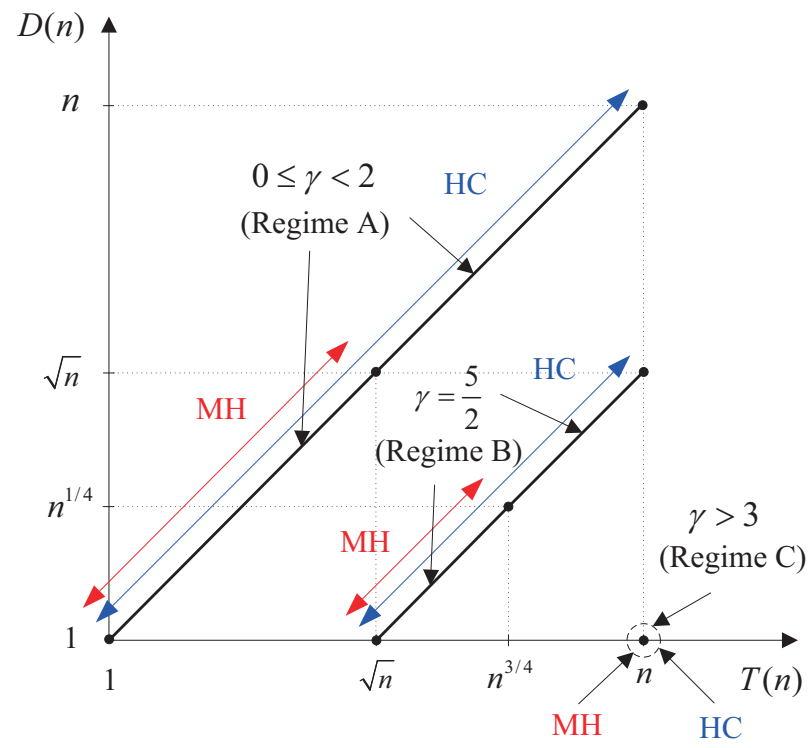

Fig. 2. The throughput-delay trade-off in a dense network when $q=\Theta(1)$. Red and blue arrows denote the trade-offs for the $\mathrm{MH}$ and network-decomposed HC protocols, respectively. Black lines indicate the best trade-offs achieved by one of these two protocols. The factor of $\log n$ is omitted for simplicity.

Theorem 2: The throughput-delay trade-off for the network-decomposed HC protocol in a wireless dense network adopting the social formation model is given by

$$
(T(n), D(n))= \begin{cases}\left(\Theta\left(n^{b-\epsilon}\right), \Theta\left(n^{b+\epsilon}\right)\right) & \text { for Regime A } \\ \left(\Theta\left(n^{b-(\gamma-2)(b-1)-\epsilon}\right), \Theta\left(n^{(3-\gamma) b+\epsilon}\right)\right) & \text { for Regime B } \\ \left(\Theta\left(n^{1-\epsilon}\right), \Theta\left(n^{\epsilon}\right)\right) & \text { for Regime C }\end{cases}
$$

for an arbitrarily small $\epsilon>0$, where $0 \leq b<1$.

Proof: See Appendix A.

From Theorem 2, the following insightful observations are found according to each operating regime. In Regime A, it is shown that the throughput-delay trade-off derived along with the social formation model is the same as that with no social behavior. In other words, the network behaves as if there is no social group when the social group density $\gamma$ is small. In Regime $\mathrm{B}$, as $\gamma$ increases, the throughput is improved and the delay is reduced. In Regime $\mathrm{C}$, the maximum throughput $\Theta(n)$ is achieved with a very small delay. These observations are similar to those for the MH case. The throughput-delay trade-off for the network-decomposed $\mathrm{HC}$ protocol is illustrated in Fig. 2 (see blue arrows).

Remark 3: When $0<\gamma<2$ (Regime A), the throughput-delay trade-off for the networkdecomposed HC protocol is given by $(T(n), D(n))=\left(\Theta\left(n^{b-\epsilon}\right), \Theta\left(n^{b+\epsilon}\right)\right)$ for $0 \leq b<1$. In Regime B, the trade-off achieved by the network-decomposed HC protocol for $\gamma=\frac{5}{2}$ (as an exemplary value of $\gamma)$ is given by $(T(n), D(n))=\left(\Theta\left(n^{(b+1) / 2-\epsilon}\right), \Theta\left(n^{b / 2+\epsilon}\right)\right)$ for $0 \leq b<1$. When $\gamma>3$ (Regime C), the trade-off for the network-decomposed HC protocol is given by $(T(n), D(n))=\left(\Theta\left(n^{1-\epsilon}\right), \Theta\left(n^{\epsilon}\right)\right)$. From Fig. 2, it is seen that for $0 \leq b \leq \frac{1}{2}$, performance on the trade-off for the $\mathrm{MH}$ and network-decomposed HC protocols is the same, but for $\frac{1}{2}<b<1$, the network-decomposed HC protocol has a higher throughput than that of the $\mathrm{MH}$ protocol at the expense of an increased delay. In the dense network, the best trade-offs can thus be achieved by the network-decomposed HC protocol and are depicted by black lines in Fig. 2 . 
Remark 4: To better understand the above trade-off between throughput and delay for the network-decomposed HC protocol in the dense network, we express $T(n)$ as a function of $D(n)$ as follows:

$$
T(n)= \begin{cases}\Theta\left(D(n) n^{-\epsilon}\right) & \text { for Regime A } \\ \Theta\left(D(n) n^{\gamma-2-\epsilon}\right) & \text { for Regime B } \\ \Theta\left(D(n) n^{1-\epsilon}\right) & \text { for Regime C }\end{cases}
$$

for an arbitrarily small $\epsilon>0$. It is observed that the throughput-delay trade-off in (9) for the network-decomposed $\mathrm{HC}$ protocol is the same as (7) for the $\mathrm{MH}$ protocol within a factor of $n^{\epsilon}$ as long as the delay $D(n)$ scales up to its maximum achieved by $\mathrm{MH}$ (refer to Fig. 2). It means that the inherent relation between the two protocols with respect to the throughputdelay trade-off remains the same, but a general trade-off can be achieved by incorporating the results for the network-decomposed HC protocol.

\section{B. Throughput-Delay Trade-off in Extended Networks}

We next show the throughput-delay trade-off of an extended network. The trade-off achieved by the $\mathrm{MH}$ protocol is first presented in the following theorem.

Theorem 3: The throughput-delay trade-off for the $\mathrm{MH}$ protocol in a wireless extended network adopting the social formation model is given by

$$
(T(n), D(n))= \begin{cases}\left(\Theta\left(\sqrt{\frac{n}{a(n)^{\alpha+1}}}\right), \Theta\left(\sqrt{\frac{n}{a(n)}}\right)\right) & \text { for Regime A } \\ \left.\Theta\left(\left(\frac{n}{\log n}\right)^{\frac{\gamma}{2}-1} \sqrt{\frac{n}{a(n)^{\alpha+1}}}\right), \Theta\left(\left(\frac{\log n}{n}\right)^{\frac{\gamma}{2}-1} \sqrt{\frac{n}{a(n)}}\right)\right) & \text { for Regime B } \\ \left(\Theta\left(\frac{n}{\sqrt{a(n)^{\alpha+1} \log n}}\right), \Theta\left(\sqrt{\frac{\log n}{a(n)}}\right)\right) & \text { for Regime C }\end{cases}
$$

where the area of each square cell is given by $a(n)=\Omega(\log n)$.

Proof: Similarly as in the proof of Theorem 1, the throughput-delay trade-off for the $\mathrm{MH}$ protocol in an extended network can easily be proved by computing the received signal power that is expressed in a different manner due to the power limitation as well as using the fact that the area of each cell, $a(n)$, is increased by a factor of $n$ compared to a dense network. Since the received signal-to-noise ratio (SNR) for the signal transmitted from the nearest-neighbor cell scales as $\left(\frac{1}{\sqrt{a(n)}}\right)^{\alpha}$, we obtain the throughput result in (10) by using (4), which completes the proof of this theorem.

In the extended network, the throughput-delay trade-off for the $\mathrm{MH}$ protocol is illustrated in Fig. 3 when $q=\Theta(1)$ and $\alpha \in\{2,3\}$. The red arrows in the figure represent the throughputdelay scaling results achieved by the MH protocol according to different values of $\gamma$.

Remark 5: To better understand the above trade-off between throughput and delay for the $\mathrm{MH}$ protocol in the extended network, we express $T(n)$ as a function of $D(n)$ as follows:

$$
T(n)= \begin{cases}\Theta\left(D(n) a(n)^{-\alpha / 2}\right) & \text { for Regime A } \\ \Theta\left(D(n) n^{\gamma-2-\epsilon} a(n)^{-\alpha / 2}\right) & \text { for Regime B } \\ \Theta\left(D(n) n^{1-\epsilon} a(n)^{-\alpha / 2}\right) & \text { for Regime C }\end{cases}
$$

for an arbitrarily small $\epsilon>0$. Compared to the dense network case (see Remark 2), the throughput $T(n)$ given the delay $D(n)$ is reduced by a factor of $a(n)^{-\alpha / 2}$, which corresponds to the amount of SNR loss. 


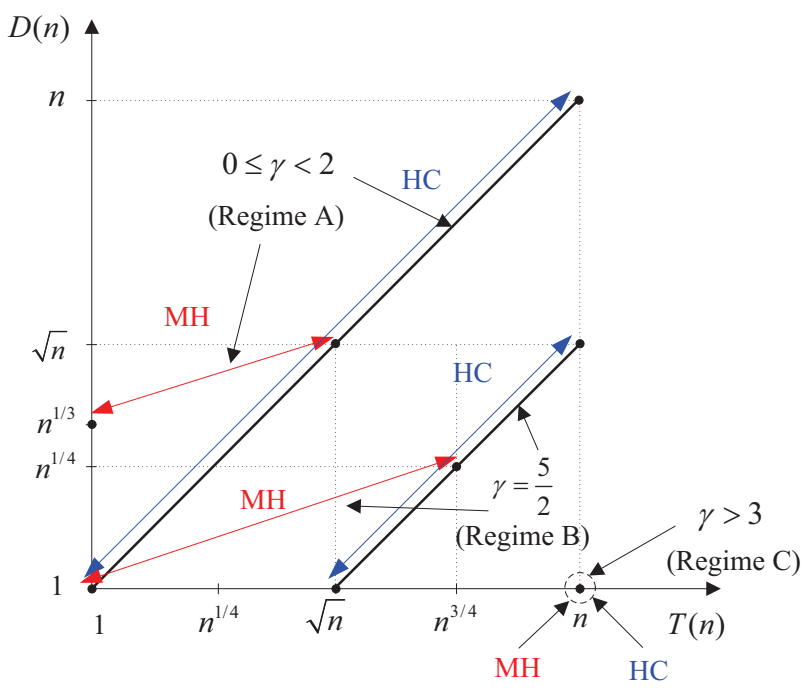

(a) $\alpha=2$

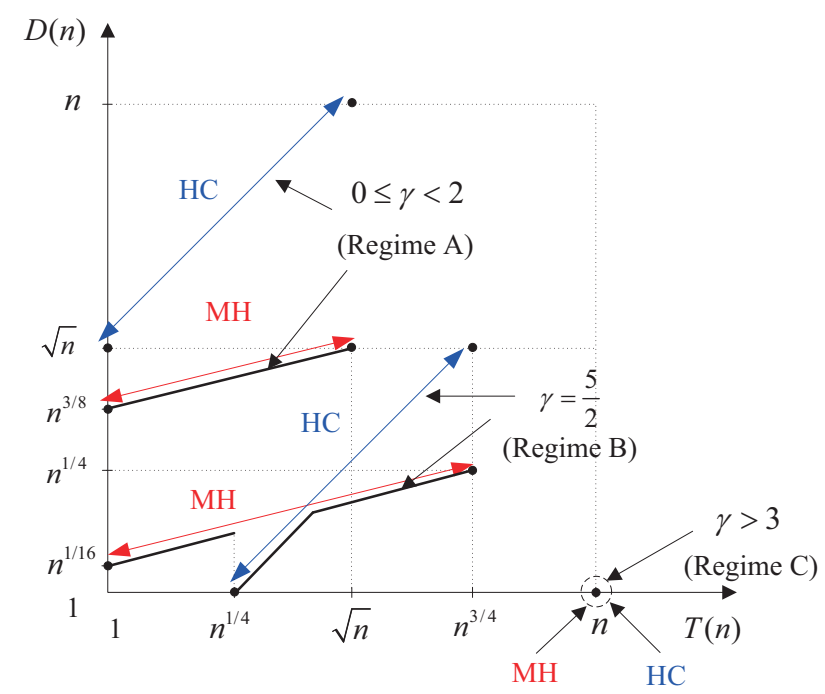

(b) $\alpha=3$

Fig. 3. The throughput-delay trade-off in an extended network when $q=\Theta(1)$. Red and blue arrows denote the trade-offs for the $\mathrm{MH}$ and network-decomposed $\mathrm{HC}$ protocols, respectively. Black lines indicate the best trade-offs achieved by one of these two protocols. The factor of $n^{\epsilon}$ is omitted for simplicity.

Now, let us turn to summarizing our main results for an extended network when the proposed network-decomposed HC protocol is employed.

Theorem 4: The throughput-delay trade-off for the network-decomposed HC protocol in a wireless extended network adopting the social formation model is given by

$$
(T(n), D(n))= \begin{cases}\left(\Theta\left(n^{b-\alpha / 2+1-\epsilon}\right), \Theta\left(n^{b+\epsilon}\right)\right) & \text { for Regime A } \\ \left(\Theta\left(n^{(b-\alpha / 2)(3-\gamma)+1-\epsilon}\right), \Theta\left(n^{(3-\gamma) b+\epsilon}\right)\right) & \text { for Regime B } \\ \left(\Theta\left(n^{1-\epsilon}\right), \Theta\left(n^{\epsilon}\right)\right) & \text { for Regime C }\end{cases}
$$

for an arbitrarily small $\epsilon>0$, where $0 \leq b<1$.

Proof: See Appendix B,

In contrast to the results for the dense network in (8), the throughput scaling achieved by the network-decomposed HC protocol in the extended network depends highly on the path- 
loss exponent $\alpha$ in Regimes A and B (i.e., the low and medium social group density regimes, respectively), while the delay scaling is the same for both dense and extended networks. This is because in the above two regimes, a bursty transmission whose duration depends on $\alpha$ is needed due to the power limitation, which will be explained in detail in Section IV-C.

Remark 6: From Theorem 4, $T(n)$ for the network-decomposed HC ptorocol in an extended network is expressed as a function of $D(n)$ as follows:

$$
T(n)= \begin{cases}\Theta\left(D(n) n^{-\alpha / 2+1-\epsilon}\right) & \text { for Regime A } \\ \Theta\left(D(n) n^{1+\alpha(\gamma-3) / 2-\epsilon}\right) & \text { for Regime B } \\ \Theta\left(D(n) n^{1-\epsilon}\right) & \text { for Regime C }\end{cases}
$$

for an arbitrarily small $\epsilon>0$.

In the extended network, the throughput-delay trade-off for the network-decomposed HC protocol is also illustrated in Fig. 3 when $q=\Theta(1)$ and $\alpha \in\{2,3\}$ (see blue arrows), and the following interesting observations in comparison with the results for the $\mathrm{MH}$ protocol are made according to each operating regime.

Remark 7: First, it is shown in Fig. 3 that the trade-offs for the $\mathrm{MH}$ and networkdecomposed HC protocols are improved as the social group density $\gamma$ increases as in the dense network configuration. We now recall that in the extended network, the throughput for a given delay depends on the path-loss exponent $\alpha$, unlike the dense network case. When $\alpha=2$, the trade-off achieved by the network-decomposed HC protocol is always superior to or equal to that of the $\mathrm{MH}$ protocol for all the operating regimes. On the other hand, as $\alpha$ increases, there exists operating regimes such that the $\mathrm{MH}$ protocol is dominant. More precisely, in Regime $\mathrm{A}$, it is not difficult to show that if $\alpha>1+\sqrt{3}$, then the MH protocol outperforms the network-decomposed HC protocol, and there is a crossover between two lines achieved by both protocols otherwise. In Regime $\mathrm{B}$, the $\mathrm{MH}$ protocol is superior to the network-decomposed $\mathrm{HC}$ protocol if

$$
\gamma \leq \frac{3 \alpha^{2}-2 \alpha-6}{\alpha^{2}-2}
$$

Otherwise, there is a crossover between two lines achieved by both protocols, where the network-decomposed $\mathrm{HC}$ protocol has better trade-off performance in the low throughput regime as depicted in Fig. 3 (b). In Regime $\mathrm{C}$, both $\mathrm{MH}$ and network-decomposed HC protocols achieve the best throughput of $\Theta(n)$ with a very small delay of $\Theta(1)$ in which the social group density $\gamma$ becomes high, as in the dense network case.

\section{Throughput-Delay Trade-off For Network-Decomposed HC}

In this section, we shall first introduce the network-decomposed HC protocol so that the network under our social formation model operates suitably, and then derive its throughputdelay trade-off in a dense network. The trade-off achieved by the network-decomposed HC protocol in an extended network is also shown through network transformation.

\section{A. Conventional HC Protocol}

We will first briefly explain the original HC protocol [30] and its modified one [42] in an ad hoc network. It will be shown how the $\mathrm{HC}$ protocol is further modified to work properly for our network with the distance-based social formation model in the subsequent subsections. In the original $\mathrm{HC}$ protocol [30], packets are transmitted through the following three phases:

- The network is divided into multiple clusters, each having $M$ nodes, where $M=O(n)$. 
- During the first phase, each source distributes its $M$ bits to other nodes in the same cluster, one bit for each node.

- During the second phase, a long-range multiple-input multiple-out (MIMO) transmission is then performed between two clusters having a source and its destination, one at a time.

- During the last phase, each node in a cluster quantizes its received observations and delivers the quantized data to the rest of nodes in the same cluster. Each destination can decode its packets by collecting all quantized observations.

When each node transmits data within its cluster, another smaller-scaled cooperation can be applied in the same manner by dividing each cluster into smaller ones. By recursively applying this procedure, it is possible to establish the hierarchical strategy in the network. One drawback of the original HC protocol [30] is that it requires an extremely large bulk-size $\Theta\left(n^{\frac{h}{2}}\right)$, i.e., the minimum number of bits that should be transmitted between each S-D pair is large, where $h>0$ is the hierarchy level. Due to a large bulk-size and inefficient scheduling, the delay scaling of the $\mathrm{HC}$ protocol can be much worse than that of the $\mathrm{MH}$ protocol. In order to improve the delay performance, the original $\mathrm{HC}$ protocol was modified in [42] by reducing the bulk-size and enhancing scheduling. Using the modified $\mathrm{HC}$ protocol [42], the throughput-delay trade-off is given by

$$
(T(n), D(n))=\Theta\left(n^{b} / \log n, n^{b} \log n\right)
$$

where $0 \leq b<1$. The parameter $b$ depends on the size of a cluster with $0 \leq b \leq \frac{h}{h+1}$.

\section{B. Network-Decomposed HC Protocol}

Both the original HC protocol [30] and its modified one [42] were designed assuming that the geographic distance of between a source node and its destination node scales as $O(1)$ in a dense network due to the random S-D pairings. Since the distance of an S-D pair in our network may scale at a lower rate than that of the network size depending on the parameters of the social formation model, the HC protocols in [30], [42] may not work effectively. This motivates us to further modify the $\mathrm{HC}$ protocol in [42] by taking into account the notion of network decomposition as in the following.

1) Network Decomposition: To fully exploit the characteristics of our distance-based social formation model, we modify the $\mathrm{HC}$ protocol so that the reduced distance of an S-D pair can be carefully incorporated into the protocol design. Owing to the central processor that leverages geo-information of nodes, it is possible to divide a network into multiple nonoverlapping subnetworks, each of which operates using the existing $\mathrm{HC}$ protocol in [42] with a reduced network size accordingly. When we denote $d_{s, v}$ as the distance between a source $s$ and its destination $v$, we choose the side length $l(n)$ of a subnetwork as $\mathbb{E}\left[d_{s, v}\right] n^{\epsilon}$ for an arbitrarily small $\epsilon>0$ so that $s$ and $v$ can coexist inside one subnetwork w.h.p., since the distance between an S-D pair does not scale at a faster rate than $\mathbb{E}\left[d_{s, v}\right]$ within a factor of $n^{\epsilon}$ w.h.p. (refer to Lemma 2). The subnetworks of constant side length

$$
l(n)=\mathbb{E}\left[d_{s, v}\right] n^{\epsilon}
$$

having S-D pairs are illustrated in Fig. 4. There are $m:=n l^{2}(n)$ nodes on average in each subnetwork, where the HC protocol in [42] is employed within each subnetwork.

2) Edge Node Problem and Protocol Refinement: When the whole network is decomposed into multiple subnetworks as in Section IV-B1, there may exist some sources in a subnetwork, whose destinations are outside of the subnetwork, as depicted by red circles in Fig. 4. Packets 


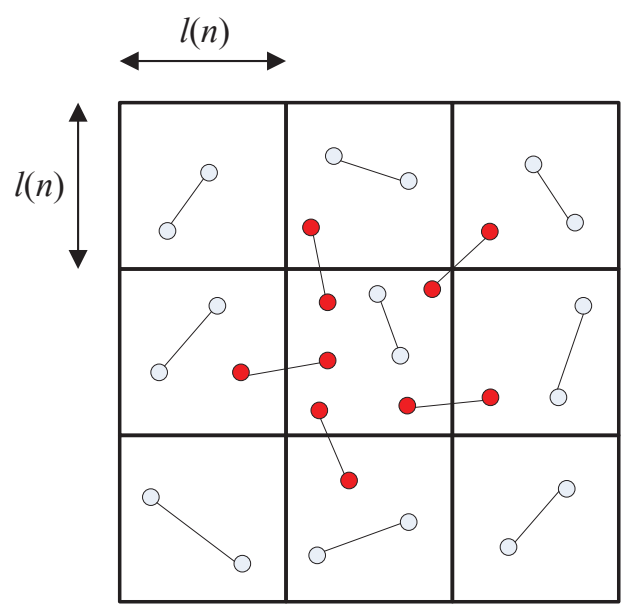

Fig. 4. The illustration of subnetworks for the network-decomposed $\mathrm{HC}$ protocol, where a subnetwork is depicted as a box, a source or destination node is denoted by a circle, and the line between two circles indicates the relationship of S-D pairings. The red circle represents the node whose transmission is performed across the adjacent subnetwork.

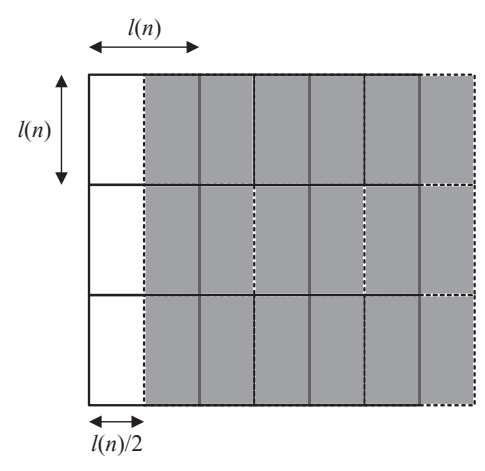

(a) Shifted to the right by $l(n) / 2$

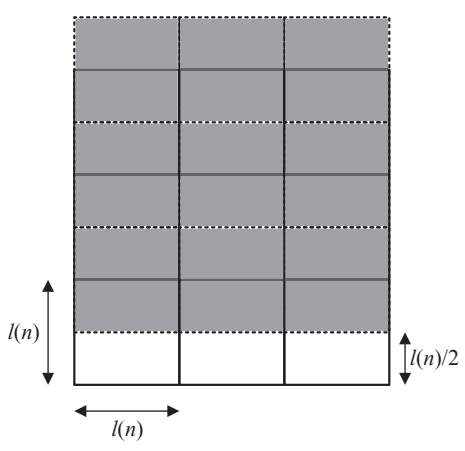

(b) Shifted up by $l(n) / 2$

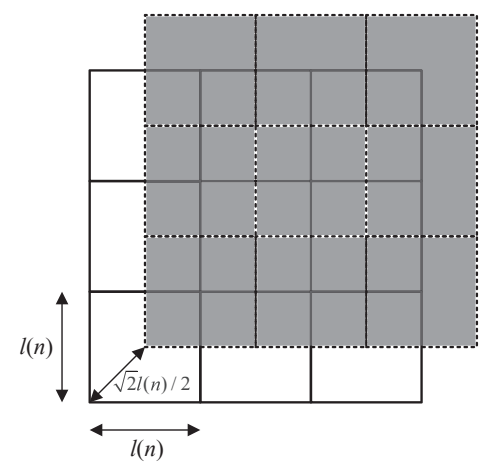

(c) Shifted diagonally by $\sqrt{2} l(n) / 2$

Fig. 5. The 4-TDMA operation of our network-decomposed HC protocol.

of these sources in the subnetwork cannot be delivered to the corresponding destinations using the existing $\mathrm{HC}$ framework that should be employed only inside each subnetwork. In order to solve this edge node problem, we apply a 4-TDMA operation with which all the subnetworks are shifted to the right, shifted up by $l(n) / 2$, and shifted diagonally by $\sqrt{2} l(n) / 2$ in consecutive time slots (see Fig. 5). By doing so, the packets of the vast majority of S-D pairs can be successfully delivered in one of time slots. Note that this subnetwork shift based on the 4-TDMA strategy does not fundamentally change the throughput and delay scaling. In each time slot, the subnetworks operate in parallel using the HC protocol with $\Theta(m)$ nodes in each subnetwork.

3) Network-Decomposed HC Protocol: We assume that packets are conveyed by the networkdecomposed HC protocol only when the geographic distance between a source node and its destination node is shorter than $\Theta(l(n))$ without affecting the overall throughput-delay tradeoff. By recalling Lemma 2, we note that the average number of S-D pairs whose distance is less than $\Theta(l(n))$ is given by $\Theta(n)$ (refer to Remark 9 for more details). According to Sections IV-B1 and IV-B2, the proposed network-decomposed HC protocol is described as follows:

- Step 1) Our ad hoc network is divided into multiple non-overlapping subnetworks, each 
of which has size $l(n) \times l(n)$.

- Step 2) The HC protocol in [42] is employed in each subnetwork.

- Step 3) All the subnetworks are shifted to the right by $l(n) / 2$ compared to their original positions, and the HC protocol is employed in each shifted subnetwork.

- Step 4) All the subnetworks are shifted up by $l(n) / 2$ compared to the original positions, and the HC protocol is employed in each shifted subnetwork.

- Step 5) All the subnetworks are shifted diagonally by $\sqrt{2} l(n) / 2$ compared to the original positions, and the HC protocol is employed in each shifted subnetwork.

- Steps 2), 3), 4), and 5) are repeated in a TDMA manner.

Under the network-decomposed HC protocol, the throughput-delay trade-off of a wireless dense network adopting the social formation model is given by the expression in (8) (refer to Theorem 2). It is shown that the throughput-delay trade-off for the network-decomposed HC protocol is improved as the social group density $\gamma$ increases, since the number of subnetworks that are activated in parallel becomes large with increasing $\gamma$.

Remark 8: In the original HC protocol, the average per-node transmit power required to run the HC protocol in dense networks is $\frac{P}{n}$ but not $P$. This is because when the $\Theta(n) \times \Theta(n)$ long-range MIMO transmission is performed at the top level of the hierarchy, the average distance between two clusters (i.e., the transmitting nodes and the receiving nodes) is $O(1)$ and an array gain of $n$ can be obtained. On the other hand, when the network-decomposed $\mathrm{HC}$ protocol is employed in our dense network, the average per-node transmit power becomes

$$
\frac{P}{m} l(n)^{\alpha}=\frac{P}{n} l(n)^{\alpha-2}
$$

due to the fact that the network-decomposed HC protocol operates within a subnetwork of $m$ nodes and the average distance between two clusters is given by $O(l(n))$.

Remark 9: Our network-decomposed HC protocol operates within each subnetwork in parallel. However, from Lemma 2, there exists a non-zero probability that a relatively small fraction of S-D pairs are further apart than the size of the subnetwork. In our work, we assume that the packets of such S-D pairs are conveyed by the MH protocol. It is not difficult to show that the resulting throughput-delay trade-off does not change at all by performing $\mathrm{MH}$ for such pairs.

\section{Extended Network Configuration}

In the previous subsection, we have focused on the analysis for a dense network of unit area. We now turn to analyzing an extended network of unit node density, whose size is $\sqrt{n} \times \sqrt{n}$. Since the distance between a source and its destination is increased by a factor of $\sqrt{n}$ in the extended network, the received signal power will be decreased by a factor of $n^{\alpha / 2}$. Hence, the extended network of size $\sqrt{n} \times \sqrt{n}$ can be treated as a dense network of size $1 \times 1$ with the reduced average per-node transmit power constraint of $\frac{P}{n^{\alpha / 2}}$. Thus, if the average per-node transmit power required to perform the network-decomposed HC protocol in the dense network is bounded by $\frac{P}{n^{\alpha / 2}}$, then the same throughput-delay trade-off for the network-decomposed HC protocol can be achieved as in the dense network case. Since the average per-node transmit power depends on side length $l(n)$ in (13), which varies according to the operating regimes, we compare the average per-node transmit power with $\frac{P}{n^{\alpha / 2}}$ in each regime as follows. Note that this network transformation along with scaling parameters $\gamma$ and $\alpha$ is not a straightforward extension of [30].

In Regime A (i.e., the low social group density regime), using Lemma 1, (12), and (13), the average per-node transmit power for the network-decomposed $\mathrm{HC}$ protocol becomes $\frac{P}{n^{1-\epsilon}}$ in 
our dense network. In order to satisfy the equivalent power constraint $\frac{P}{n^{\alpha / 2}}$, we need a bursty transmission strategy similarly as in [30]. Specifically, the network-decomposed HC protocol is performed during a fraction $\frac{1}{n^{\alpha / 2-1+\epsilon}}$ of the time with per-node power $\frac{P}{n^{1-\epsilon}}$ and remains silent for the rest of the time. Then, the throughput is reduced to $T(n)=\Theta\left(n^{b-\alpha / 2+1-\epsilon}\right)$ while the delay is the same as that in the dense network. In Regime B (i.e., the medium social group density regime), the average per-node transmit power required to run the network-decomposed HC protocol becomes $\frac{P}{n^{1+(\gamma / 2-1)(\alpha-2)-\epsilon}}$ in the dense network due to the fact that $l(n)$ depends on the social group density $\gamma$. In the regime, we need to use a bursty transmission that runs the network-decomposed HC protocol during a fraction $\frac{1}{n^{(1-\alpha / 2)(\gamma-3)+\epsilon}}$ of the time, resulting in $T(n)=\Theta\left(n^{b-(\gamma-2)(b-1)-(1-\alpha / 2)(\gamma-3)-\epsilon}\right)$ with the same delay as in the dense network. In Regime C (i.e., the high social group density regime), the average per-node transmit power is $\frac{P}{n^{\alpha / 2-\epsilon}}$ in the dense network due to $l(n)=\Theta\left(n^{-1 / 2+\epsilon}\right)$. Hence, the network-decomposed HC protocol runs during a fraction $n^{-\epsilon}$. The throughput is then given by $T(n)=\Theta\left(n^{1-\epsilon}\right)$ with the same delay as in the dense network.

Based on this bursty modification according to each operating regime, we can establish the throughput-delay trade-off in (11) for the network-decomposed HC protocol in the extended network with our social formation model (see Theorem 4). Compared to the dense network case, the throughput-delay trade-offs in Regimes A and B are degraded as the path-loss exponent $\alpha$ increases since the extended network is power-limited in these two regimes. On the other hand, in Regime $\mathrm{C}$, the network is not power-limited due to the high social group density, where the same throughput-delay trade-off can be achieved as in the dense network case.

\section{NUMERicAl EVALUATION}

In this section, we perform computer simulations according to finite values of $n$ to validate that our analytical results show trends consistent with numerical results. We evaluate the performance of the network-decomposed HC protocol in terms of the total throughput $T(n)$, which can be computed as the sum of the transmission rates in parallel across all subnetworks. The capacity of each subnetwork is bounded by the rate of long-range MIMO transmission between two clusters having a source and its destination in a subnetwork. A sufficient number of nodes $(n=256)$ are deployed so that a large-scale network is suitably modeled in practice. It is assumed that the network size is given by $100 \times 100\left(\mathrm{~m}^{2}\right)$. All the transmit power is set to the same value as the noise variance.

Now, we turn to describing how to generate multiple network configurations, each of which has different numbers of subnetworks, depending on values of the social group density $\gamma$. Let $b_{\gamma}$ denote the parameter $b$ in Theorems 2 and 4 for given $\gamma$. In our simulations, we set $b_{2}=\frac{1}{4}$. Then, from the results of the two theorems, we have $b_{\gamma}=\frac{b_{2}}{3-\gamma}=\frac{1}{4(3-\gamma)}$ to ensure that the delays for all values of $\gamma$ are the same in order sense. From the fact that the side length of a subnetwork in (12) is given by $\mathbb{E}\left[d_{s, v}\right]$ in (3) for given $\gamma$, it follows that four values $2,2.25$, $2+\frac{\log _{2} 3}{4}$, and 2.5 for $\gamma$ are chosen to generate such network configurations that have 1,4 , 9 , and 16 subnetworks, respectively. Note that $\gamma=2$ corresponds to the baseline employing the conventional $\mathrm{HC}$ protocol since there exists a single subnetwork.

In Fig. 6, the total throughput versus the social group density $\gamma$ is illustrated, where $\alpha \in\{2,2.5,3\}$. From the figure, the following observations are made under our simulation environments: the throughput performance is degraded with increasing path-loss exponent $\alpha$ due to more severe path-loss attenuation; the total throughput is gradually enhanced as $\gamma$ increases up to 2.4; and the total throughput is greatly improved when $\gamma=2.5$ since 


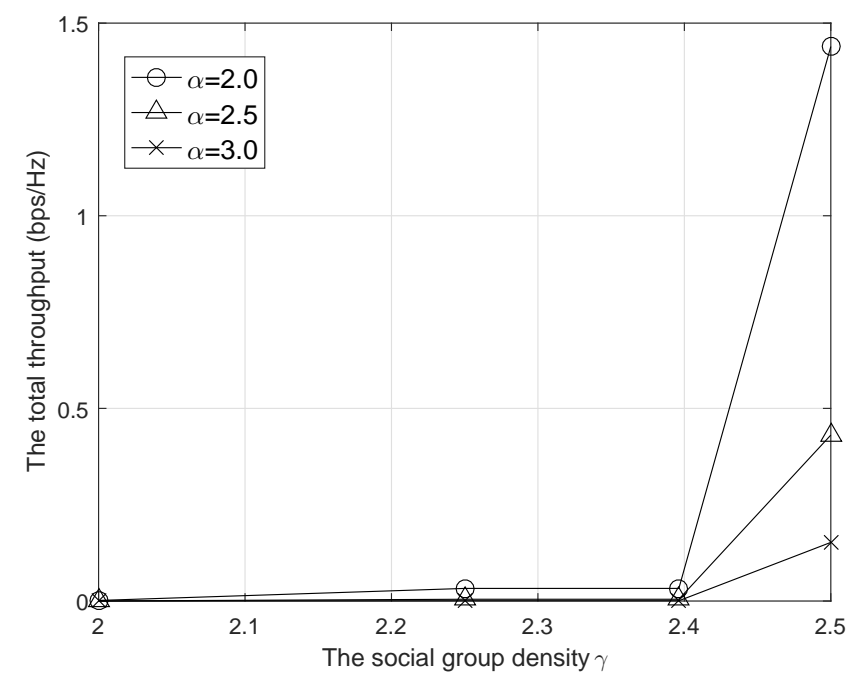

Fig. 6. The total throughput according to the social group density $\gamma$, where $\alpha \in\{2.0,2.5,3.0\}$.

the number of subnetworks is largely increased as the distance between a source and its destination, $\mathbb{E}\left[d_{s, v}\right]$, decreases with respect to $\gamma$. This result implies that the social behavior among nodes in ad hoc networks has a significant impact on the throughput performance.

\section{CONCLUding REMARKS}

By introducing a new $\mathrm{HC}$ protocol, we completely characterized the general throughputdelay trade-off of dense and extended ad hoc networks with the distance-based social formation model parameterized by the social group density $\gamma$ and the number of social contacts per node, $q$, where a source selects one of its social contacts as its destination uniformly at random. More precisely, we proposed the network-decomposed HC protocol so that the networks operate properly under our social formation model in terms of maximizing the throughput-delay trade-off. To more concisely show our main results, we also identified three operating regimes on the throughput-delay trade-off with respect to $\gamma$ and $q$. In the dense network, we showed that when $\gamma$ is small, the throughput-delay trade-off is the same as the non-social behavior scenario; on the other hand, when $\gamma$ increases, the throughput-delay trade-off is significantly improved; and when $\gamma$ becomes large, the maximum throughput $\Theta(n)$ can be achieved via a single-hop transmission, leading to the delay of $\Theta(1)$. In addition, we analyzed the corresponding throughput-delay trade-off in the extended network through the nontrivial network transformation strategy, and investigated the operating regimes such that the network-decomposed $\mathrm{HC}$ protocol outperforms the $\mathrm{MH}$ protocol according to parameters $\gamma$ and $\alpha$. Suggestions for further research include analyzing performance on the throughput and delay when the size of social groups follows a well-known Zipf's distribution instead of assuming the same size of all social groups. Another interesting direction is to investigate the effect of queuing delay on the throughput-delay trade-off for the network-decomposed HC protocol in ad hoc networks with social relationships. 


\section{APPENDIX A \\ PROOF OF THEOREM 2}

In a dense network, the throughput-delay trade-off achieved by the network-decomposed $\mathrm{HC}$ protocol in a subnetwork consisting of $m=n l^{2}(n)$ nodes is given by [42]

$$
(T(n), D(n))=\Theta\left(m^{b} / \log m, m^{b} \log m\right),
$$

where $0 \leq b<1$. Since there are $\frac{n}{m}$ subnetworks over the whole network, the aggregate throughput is $\frac{n}{m}$ times the throughput of each subnetwork while the delay of the network remains the same due to the fact that all the subnetworks operate in parallel. Hence, the throughput-delay trade-off of the network is given by

$$
(T(n), D(n))=\Theta\left(m^{b-1} n / \log m, m^{b} \log m\right),
$$

where $0 \leq b<1$. Substituting $m=n \mathbb{E}^{2}\left[d_{s, v}\right] n^{2 \epsilon}$ into A.1. where $d_{s, v}$ denotes the distance between a source $s$ and its destination $v$, we have

$$
\begin{gathered}
(T(n), D(n))=\Theta\left(n^{b}\left(\mathbb{E}\left[d_{s, v}\right] n^{\epsilon}\right)^{2 b-2} / \log \left(n\left(\mathbb{E}\left[d_{s, v}\right] n^{\epsilon}\right)^{2}\right),\right. \\
\left.n^{b}\left(\mathbb{E}\left[d_{s, v}\right] n^{\epsilon}\right)^{2 b} \log \left(n\left(\mathbb{E}\left[d_{s, v}\right] n^{\epsilon}\right)^{2}\right)\right) .
\end{gathered}
$$

In what follows, we derive the throughput and the delay according to each operating regime.

\section{A. Regime A}

For $q(n)=\omega(1)$, since $\mathbb{E}\left[d_{s, v}\right]=\Theta(1)$ from Lemma 1, the throughput $T(n)$ and the delay $D(n)$ are given by

$$
\begin{aligned}
(T(n), D(n)) & =\Theta\left(n^{b+\epsilon(2 b-2)} / \log n^{1+2 \epsilon}, n^{b+2 \epsilon b} \log n^{1+2 \epsilon}\right) \\
& =\Theta\left(n^{b-\epsilon}, n^{b+\epsilon}\right) .
\end{aligned}
$$

For $q=\Theta(1)$ and $0 \leq \gamma<2$, we have the same result as above due to $\mathbb{E}\left[d_{s, v}\right]=\Theta(1)$.

\section{B. Regime B}

For $q=\Theta(1)$ and $2 \leq \gamma \leq 3$, it follows that $\mathbb{E}\left[d_{s, v}\right]=\Theta\left(\left(\frac{\log n}{n}\right)^{\frac{\gamma}{2}-1}\right)$ from Lemma 1, Thus, we have

$$
\begin{aligned}
& T(n)=\Theta\left(n^{b}\left(\left(\frac{\log n}{n}\right)^{\frac{\gamma}{2}-1} n^{\epsilon}\right)^{2 b-2} \frac{1}{\log \left(n\left(\left(\frac{\log n}{n}\right)^{\frac{\gamma}{2}-1} n^{\epsilon}\right)^{2}\right)}\right), \\
& D(n)=\Theta\left(n^{b}\left(\left(\frac{\log n}{n}\right)^{\frac{\gamma}{2}-1} n^{\epsilon}\right)^{2 b} \log \left(n\left(\left(\frac{\log n}{n}\right)^{\frac{\gamma}{2}-1} n^{\epsilon}\right)^{2}\right)\right),
\end{aligned}
$$

which can be simplified to $(T(n), D(n))=\left(\Theta\left(n^{b-(\gamma-2)(b-1)-\epsilon}\right), \Theta\left(n^{(3-\gamma) b+\epsilon}\right)\right)$. 


\section{Regime C} have

For $q=\Theta(1)$ and $\gamma>3$, it follows that $\mathbb{E}\left[d_{s, v}\right]=\Theta\left(\sqrt{\frac{\log n}{n}}\right)$ from Lemma 11. Thus, we

$$
\begin{aligned}
& T(n)=\Theta\left(n^{b}\left(\sqrt{\frac{\log n}{n}} n^{\epsilon}\right)^{2 b-2} \frac{1}{\log \left(n\left(\sqrt{\frac{\log n}{n}} n^{\epsilon}\right)^{2}\right)}\right) \\
& D(n)=\Theta\left(n^{b}\left(\sqrt{\frac{\log n}{n}} n^{\epsilon}\right)^{2 b} \log \left(n\left(\sqrt{\frac{\log n}{n}} n^{\epsilon}\right)^{2}\right)\right),
\end{aligned}
$$

which can be rewritten as $(T(n), D(n))=\left(\Theta\left(n^{1-\epsilon}\right), \Theta\left(n^{\epsilon}\right)\right.$. In consequence, the throughputdelay trade-off for the network-decomposed HC protocol in the dense network is given by the expression in (8), which completes the proof of this theorem.

\section{APPENDIX B \\ Proof OF THEOREM 4}

In an extended network, we derive the throughput-delay trade-off achieved by the networkdecomposed $\mathrm{HC}$ protocol according to each operating regime in the following. Based on the power limitation argument in Section IV-C, the extended network is equivalent to the dense network with the average per-node transmit power constraint of $\frac{P}{n^{\alpha / 2}}$. Note that from (13), the average per-node transmit power required for the network-decomposed HC protocol in the dense network is $\frac{P}{m} l(n)^{\alpha}=\frac{P}{n} l(n)^{\alpha-2}$ (but not $P$ ). We will compare this required power with the average per-node power constraint of $\frac{P}{n^{\alpha / 2}}$ in each regime.

\section{A. Regime A}

For $q(n)=\omega(1)$ or $\{q=\Theta(1)$ and $0 \leq \gamma<2\}$, the network-decomposed HC protocol is performed with the average per-node transmit power constraint of $\frac{P}{n} l(n)^{\alpha-2}=\frac{P}{n}\left(n^{\epsilon}\right)^{\alpha-2}$. We use a bursty transmission strategy similarly as in [30] in order to satisfy the corresponding power constraint $\frac{P}{n^{\alpha / 2}}$, where the network-decomposed HC protocol is performed during a fraction $\frac{1}{n^{\alpha / 2-1+\epsilon}}$ of the time with per-node power $\frac{P}{n^{1-\epsilon}}$ and remains silent for the rest of the time. The throughput $T(n)$ and the delay $D(n)$ are then given by $T(n)=\Theta\left(n^{b-\alpha / 2+1-\epsilon}\right)$ and $D(n)=\Theta\left(n^{b+\epsilon}\right)$, respectively.

\section{B. Regime $B$}

For $q=\Theta(1)$ and $2 \leq \gamma \leq 3$, we use a bursty transmission that runs the networkdecomposed HC protocol during a fraction $\frac{1}{n^{(1-\alpha / 2)(\gamma-3)+\epsilon}}$ of the time. In this case, we have

$$
(T(n), D(n))=\left(\Theta\left(n^{(b-\alpha / 2)(3-\gamma)+1-\epsilon}\right), \Theta\left(n^{(3-\gamma) b+\epsilon}\right)\right) .
$$

\section{Regime $C$}

For $q=\Theta(1)$ and $\gamma>3$, we use a bursty transmission that runs the network-decomposed $\mathrm{HC}$ protocol during a fraction $n^{-\epsilon}$, resulting in $(T(n), D(n))=\left(\Theta\left(n^{1-\epsilon}\right), \Theta\left(n^{\epsilon}\right)\right)$.

In consequence, the throughput-delay trade-off for the network-decomposed HC protocol in the extended network is given by the expression in (11), which completes the proof of this theorem. 


\section{REFERENCES}

[1] B. Latané, J. H. Liu, A. Nowak, M. Bonevento, and L. Zheng, "Distance matters: Physical space and social impact," Personality and Social Psychology Bulletin, vol. 21, no. 8, pp. 795-805, Aug. 1995.

[2] J. M. Kleinberg, "Navigation in a small world," Nature, vol. 406, no. 6798, pp. 845-845, Aug. 2000.

[3] L. Backstrom, E. Sun, and C. Marlow, "Find me if you can: Improving geographical prediction with social and spatial proximity," in Proc. International Conference on World Wide Web, Raleigh, NC, Apr. 2010, pp. 61-70.

[4] D. Liben-Nowell, J. Novak, R. Kumar, P. Raghavan, and A. Tomkins, "Geographic routing in social networks," PNAS of the United States of America, vol. 102, no. 33, pp. 11623-11628, Aug. 2005.

[5] W.-Y. Shin, B. C. Singh, J. Cho, and A. M. Everett, "A new understanding of friendships in space: Complex networks meet Twitter," J. Inf. Sci., vol. 41, no. 6, pp. 751-764, Dec. 2015.

[6] H. Kwan, C. Lee, H. Park, and S. Moon, "What is twitter, a social network or a news media?" in Proc. International Conference on World Wide Web, Raleigh, NC, Apr. 2010, pp. 591-600.

[7] B. Viswanath, A. Mislove, M. Cha, and K. P. Gummadi, "On the evolution of user interaction in facebook," in Proc. ACM Workshop on Online Social Networks (WOSN), Barcelona, Spain, Aug. 2009, pp. 37-42.

[8] A. Mislove, H. Koppula, K. Gummadi, P. Druschel, and B. Bhattacharjee, "Growth of the flickr social network," in Proc. ACM Workshop on Online Social Networks (WOSN), Seattle, WA, Aug. 2008, pp. 25-30.

[9] Y. Chen, C. Zhuang, Q. Cao, and P. Hui, "Understanding cross-site linking in online social networks," in Proc. ACM Workshop on Social Network Mining and Analysis, New York City, NY, Aug. 2014, pp. 1-9.

[10] K.-C. Chen, M. Chiang, and H. V. Poor, "From technological networks to social networks," IEEE J. Select. Areas Commun., vol. 31, no. 9, pp. 548-572, Sept. 2013.

[11] X. Gong, X. Chen, K. Xing, D.-H. Shin, M. Zhang, and J. Zhang, "From social group utility maximization to persoanlized location privacy in mobile networks," IEEE/ACM Trans. Networking, vol. 25, no. 3, pp. 1703-1716, June 2017.

[12] E. Datsika, A. Antonopoulos, N. Zorba, and C.Verikoukis, "Green cooperative device-to-device communication: A social-aware perspective," IEEE Access, vol. 4, pp. 3697-3707, June 2016.

[13] S. Gitzenis, G. S. Paschos, and L. Tassiulas, "Asymptotic laws for joint content replication and delivery in wireless networks," IEEE Trans. Inform. Theory, vol. 59, no. 5, pp. 2760-2776, May 2013.

[14] A. Malik, S. H. Lim, and W.-Y. Shin, "On the effects of subpacketization in content-centric mobile networks," IEEE J. Select. Areas Commun., to appear.

[15] Y. Zhang, E. Pan, L. Song, W. Saad, Z. Dawy, and Z. Han, "Social network aware device-to-device communication in wireless networks," IEEE Trans. Wireless Commun., vol. 14, no. 1, pp. 177-190, Jan. 2015.

[16] Y. Zhao, Y. Li, Y. Cao, T. Jiang, and N. Ge, "Social-aware resource allocation for device-to-device communications underlying cellular networks," IEEE Trans. Wireless Commun., vol. 14, no. 12, pp. 6621-6634, Dec. 2015.

[17] Y. Li, T. Wu, P. Hui, D. Jin, and S. Chen, "Social-aware D2D communications: Qualitative insights and quantitative analysis," IEEE Commun. Mag., vol. 52, no. 6, pp. 150-158, June 2014.

[18] Y. Li, P. Hui, D. Jin, L. Su, and L. Zeng, "Evaluating the impact of social selfishiness on the epidemic routing in delay tolerant networks," IEEE Commun. Lett., vol. 14, no. 11, pp. 1026-1028, Nov. 2010.

[19] O. Semiari, W. Saad, S. Valentin, M. Bennis, and H. V. Poor, "Context-aware small cell networks: How social metrics improve wireless resource allocation," IEEE Trans. Wireless Commun., vol. 14, no. 11, pp. 5927-5940, Nov. 2015.

[20] M. A. Alim, T. Pan, M. Thai, and W. Saad, "Leveraging social communities for optimizing cellular device-to-device communications," IEEE Trans. Wireless Commun., vol. 16, no. 1, pp. 551-564, Jan. 2017.

[21] B. Azimdoost, H. R. Sadjadpour, and J. J. Garcia-Luna-Aceves, "Capacity of wireless networks with social behavior," IEEE Trans. Wireless Commun., vol. 12, no. 1, pp. 60-69, Jan. 2013.

[22] M. K. Kiskani, B. Azimdoost, and H. R. Sadjadpour, "Effect of social groups on the capacity of wireless networks," IEEE Trans. Wireless Commun., vol. 15, no. 1, pp. 3-13, Jan. 2016.

[23] C. Wang, L. Shao, Z. Li, L. Yang, X.-Y. Li, and C. Jiang, "Capacity scaling of wireless social networks," IEEE Trans. Parallel Distrib. Syst., vol. 26, no. 7, pp. 1839-1850, July 2015.

[24] R. Hou, Y. Cheng, J. Li, M. Sheng, and K.-S. Lui, "Capacity of hybrid wireless networks with long-range social contacts behavior," IEEE/ACM Trans. Networking, vol. 25, no. 2, pp. 834-848, Apr. 2017.

[25] P. Gupta and P. R. Kumar, "The capacity of wireless networks," IEEE Trans. Inform. Theory, vol. 46, no. 2, pp. 388-404, Mar. 2000.

[26] M. Franceschetti, O. Dousse, D. N. C. Tse, and P. Thiran, "Closing the gap in the capacity of wireless networks via percolation theory," IEEE Trans. Inform. Theory, vol. 53, no. 3, pp. 1009-1018, Mar. 2007.

[27] P. Gupta and P. R. Kumar, "Towards an information theory of large networks: An achievable rate region," IEEE Trans. Inform. Theory, vol. 49, no. 8, pp. 1877-1894, Aug. 2003.

[28] F. Xue, L.-L. Xie, and P. R. Kumar, "The transport capacity of wireless networks over fading channels," IEEE Trans. Inform. Theory, vol. 51, no. 3, pp. 834-847, Mar. 2005.

[29] W.-Y. Shin, S.-Y. Chung, and Y. H. Lee, "Parallel opportunistic routing in wireless networks," IEEE Trans. Inform. Theory, vol. 59, no. 10, pp. 6290-6300, Oct. 2013.

[30] A. Özgür, O. Lévêque, and D. N. C. Tse, "Hierarchical cooperation achieves optimal capacity scaling in ad hoc networks," IEEE Trans. Inform. Theory, vol. 53, no. 10, pp. 3549-3572, Oct. 2007.

[31] U. Niesen, P. Gupta, and D. Shah, "On capacity scaling in arbitrary wireless networks," IEEE Trans. Inform. Theory, vol. 55, no. 9, pp. 3959-3982, Sept. 2009. 
[32] A. Zemlianov and G. de Veciana, "Capacity of ad hoc wireless networks with infrastructure support," IEEE J. Select. Areas Commun., vol. 23, no. 3, pp. 657-667, Mar. 2005.

[33] O. Dousse, P. Thiran, and M. Hasler, "Connectivity in ad-hoc and hybrid networks," in Proc. IEEE INFOCOM, New York, NY, June 2002, pp. 1079-1088.

[34] W.-Y. Shin, S.-W. Jeon, N. Devroye, M. H. Vu, S.-Y. Chung, Y. H. Lee, and V. Tarokh, "Improved capacity scaling in wireless networks with infrastructure," IEEE Trans. Inform. Theory, vol. 57, no. 8, pp. 5088-5102, Aug. 2011.

[35] M. Grossglauser and D. N. C. Tse, "Mobility increases the capacity of ad hoc wireless networks," IEEE/ACM Trans. Networking, vol. 10, no. 4, pp. 477-486, Aug. 2002.

[36] G. Zhang, Y. Xu, X. Wang, and M. Guizani, "Capacity of hybrid wireless networks with directional antenna and delay constraint," IEEE Trans. Commun., vol. 58, no. 7, pp. 2097-2106, July 2010.

[37] P. Li, C. Zhang, and Y. Fang, "The capacity of wireless ad hoc networks using directional antennas," IEEE Trans. Mobile Comput., vol. 10, no. 10, pp. 1374-1387, Oct. 2011.

[38] J. Yoon, W.-Y. Shin, and S.-W. Jeon, "Elastic routing in ad hoc networks with directional antennas," IEEE Trans. Mobile Comput., to appear.

[39] M. J. Neely and E. Modiano, "Capacity and delay tradeoffs for ad hoc mobile networks," IEEE Trans. Inform. Theory, vol. 51, no. 6, pp. 1917-1937, June 2005.

[40] A. E. Gamal, J. Mammen, B. Prabhakar, and D. Shah, "Optimal throughput-delay scaling in wireless networks-Part I: The fluid model," IEEE Trans. Inform. Theory, vol. 52, no. 6, pp. 2568-2592, June 2006.

[41] _ - "Optimal throughput-delay scaling in wireless networks-Part II: Constant-size packets," IEEE Trans. Inform. Theory, vol. 52, no. 11, pp. 5111-5116, Nov. 2006.

[42] A. Özgür and O. Lévêque, "Throughput-delay tradeoff for hierarchical cooperation in ad hoc wireless networks," IEEE Trans. Inform. Theory, vol. 56, no. 3, pp. 1369-1377, Mar. 2010.

[43] C. Manning and H. Schütze, Foundations of statistical natural language processing. MIT press, 1999.

[44] M. Franceschetti, M. D. Migliore, and P. Minero, "The capacity of wireless networks: Information-theoretic and physical limits," IEEE Trans. Inform. Theory, vol. 55, no. 8, pp. 3413-3424, Aug. 2009.

[45] S.-H. Lee and S.-Y. Chung, "Capacity scaling of wireless ad hoc network: Shannon meets Maxwell," IEEE Trans. Inform. Theory, vol. 58, no. 3, pp. 1702-1715, Mar. 2012.

[46] Y. Nebat, R. L. Cruz, and S. Bhardwaj, "The capacity of wireless networks in nonergodic random fading," IEEE Trans. Inform. Theory, vol. 55, no. 6, pp. 2478-2493, June 2009.

[47] A. Jovicic, P. Viswanath, and S. R. Kulkarni, "Upper bounds to transport capacity of wireless networks," IEEE Trans. Inform. Theory, vol. 50, no. 11, pp. 2555-2565, Nov. 2004. 